\title{
Effect of Lung Parenchyma on Bronchial Collapsibility during Maximum Expiratory Flow in Dogs
}

\author{
Hidetada Sasaki, Masao Nakamura and Tamotsu Takishima \\ The First Department of Internal Medicine, Tohoku University \\ School of Medicine, Sendai
}

\begin{abstract}
Sasaki, H., Nakamura, M, and Takishma, T. Effect of Lung Parenchyma on Maximum Expiratory Flow in Dogs. Tohoku J. exp. Med., 1976, 118 (1), 1-10 - Effect of peribronchial lung parenchyma on bronchial collapsibility during the maximum expiration was studied in the dog lung. Maximum expiratory flow volume curve (MEFVC) was obtained from dogs with intact parenchyma (I), parenchyma being dissected away from main lobar bronchi $1.5 \mathrm{~cm}$ in depth (II) and the dissected parenchyma glued to the bronchi with adhesive substance (III). Lung elastic pressure-volume curve and airway resistance were almost unchanged in the three conditions. Maximum expiratory flow $\left(\dot{V}_{\text {max }}\right)$ in condition II was considerably decreased and the ratio of decrease to the control value was high at the lower lung volumes. $\dot{V}_{\max }$ in condition III was significantly higher than the control values except at the very low lung volumes. It was proved that even if the airway down to segmental bronchi was dissected from peribronchial lung parenchyma, $\dot{V}_{\max }$ was significantly limited. It was concluded that the radial traction of peribronchial parenchyma to the bronchi reduced bronchial collapsibility considerably, and in the dissected bronchi of condition II dynamic airway collapse would occur very easily.—_ airway mechanics; check valve phenomenon
\end{abstract}

Effect of airway collapsibility on the maximum expiratory flow ( $\left.\dot{V}_{\max }\right)$ has been discussed by many investigators. Pride et al. (1967) suggested that collapsibility of the tracheobronchial tree played an important role in limiting $\dot{V}_{\max }$. Takishima and Sasaki (1972) also suggested theoretically that $\dot{V}_{\text {max }}$ might be determined as a function of both airway resistance and airway compliance. Although there is no doubt in the fact that airway collapsibility is affected by not only the compliances of bronchial wall itself but also peribronchial lung parenchyma, little is known quantitatively about the influence of lung parenchyma on the bronchial collapsibility.

Mead et al. (1970) reported from their two dimensional stress strain models that the effective distending pressure surrounding bronchi could differ systematically from transpulmonary pressure, to the extent that the bronchi were less distensible than the surrounding lung, becoming increasingly more subatmospheric than pleural pressure as transpulmonary pressure increased, depending on the pressure-volume characteristics of the bronchi and the lung parenchyma.

Received for publication, July 2, 1975. 
Recently, Takishima et al. (1975) examined the bronchial pressure-volume curves of the dog lungs, bronchi being obstructed airtight with beads, and compared the bronchial compliance curves with and without lung parenchyma. They demonstrated that the intact bronchi within the lung showed a remarkably greater resistance to collapse than the dissected bronchi, and this characteristics tended to be much more intense when lung tension was increased, suggesting that $\dot{V}_{\max }$ in dissected bronchi, or bronchi without parenchymal support, might decrease more than that of intact bronchi.

In the present study, it is examined that the parenchyma really has a force to stabilize the bronchi of excised dog lung during maximum expiratory flow maneuvers and if so, how much it does influence on them. MEFVC was studied in the excised dog lungs with intact parenchyma, without parenchyma, and with dissected parenchyma resurrounded airtight along the lobar main bronchi about $1.5 \mathrm{~cm}$ in depth.

\section{METHODS}

Three mongrel dogs weighing $12-13 \mathrm{~kg}$ were anesthetized with sodium pentobarbital and sacrificed by exsanguination after intravenous injection of heparin. The weight of exicsed lower lobes was $35 \mathrm{~g}, 36 \mathrm{~g}$, and $25 \mathrm{~g}$ in dogs Nos. 1-3, respectively. The lobar bronchus was tied to a cannula of $1.1 \mathrm{~cm}$ internal diameter and $1.5 \mathrm{~cm}$ in length and was suspended in a Lucite box. The bronchial segment distal to the cannula but not surrounded by parenchyma was kept as short as possible.

The lobar bronchus was connected to Benedict-Roth type respirometer with a linear transducer (535 DL $1000 \mathrm{Bm}$, Sanborn) in series, generating an electrical signal proportional to the volume change $(\mathrm{Vl})$. The elastic recoil pressure $(\mathrm{Pl})$ was obtained from the difference between the pressure at the endobronchial tube placed at the end of cannula and intra Lucite box pressure, connecting to differential transducer (DLPU 0.05, Nihonkohden). The volume signal was recorded on the $Y$ axis and $P l$ on the $X$ axis of the $X-Y$ recorder (7045 A Hewlett Packard). The lobe was inflated with negative pressure in the box produced by vaccum pump. After several deep ventilation inflation and deflation quasi-static pressure-volume curves in all lobes were measured during 2 min over the whole vital capacity (VC) which was defined as the volume of gas expelled from the lung when static transpulmonary pressure was reduced from 30 to $0 \mathrm{cmH}_{2} \mathrm{O}$.

Prior to measurement of resistance, the lungs were fully inflated 3 times repeatedly, and were oscillated sinusodially at a rate of 3 cycles/sec by a loudspeaker powered by a variable-frequency sine wave generator. Flow was measured with the Fleisch pneumotachograph connected to a pressure transducer (RP-2, Nihon-kohden) at the opening of lobar bronchus. Both $\mathrm{Pl}$ and flow were recorded on a penwriting oscillograph (83, San-ei). The amplified flow signal was sent to an integrator (RFJ-5, Nihon-kohden) and recorded as a volume representation on the oscillograph. Then airway resistance (Rl) was calculated graphically according to Goldman et al. (1970). Measurements were made at $\mathrm{Pl}$ of 10,5 and $2 \mathrm{cmH}_{2} \mathrm{O}$ which were varied by changing the box pressure.

To measure MEFVC, the lung was inflated to a $\mathrm{Pl}$ of $30 \mathrm{cmH}_{2} \mathrm{O}$ by a negative box pressure. A forced deflation was produced by turning a cock from negative pump to positive pump abruptly. Positive box pressure of $50 \mathrm{cmH}_{2} \mathrm{O}$ was preserved from the beginning to the end of maximum expiration (Fig. 1). Flow and volume signals, obtained from integrated flow signal, were displayed on the storage oscilloscope, respectively (5103N, Tektronix). After it was ascertained that the identical MEFVCs were obtained by repeated forced maneuvers, the last MEFVC was photographed by a polaroid camera (C5, Tektronix). 


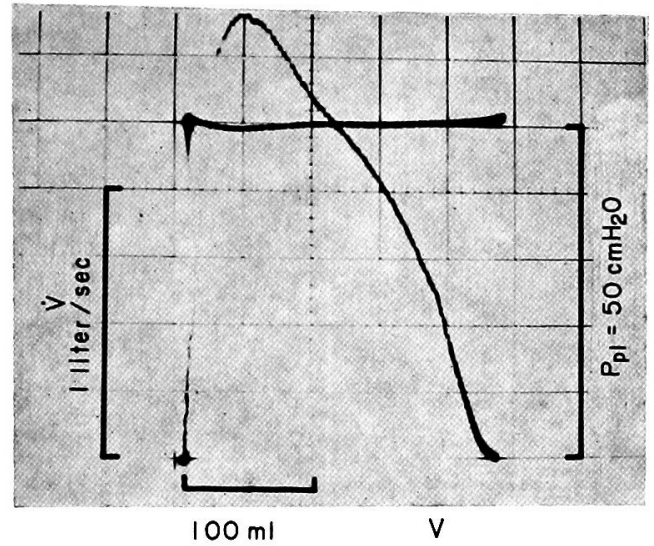

Fig. 1. MEFVC of intact bronchi which caused airleaks during parenchymal dissection. The horizontal curve showed the box pressure, that is, pleural pressure $(\mathrm{Ppl})$ of 50 $\mathrm{cmH}_{2} \mathrm{O}$ during MEFVC.

Finally, the residual lung volume was measured by water displacement technique. These measurements in series were done respectively in three different conditions as follows:

I) Control values of the intact lobe.

II) Peribronchial lung parenchyma was dissected carefully airtight with the aid of a magnifying glass from the lobar bronchi along average $1.5 \mathrm{~cm}(2.0 \sim 1.0 \mathrm{~cm})$ in length at $\mathrm{Pl}=0 \mathrm{cmH}_{2} \mathrm{O}$. Fig. 2(a) and (b) showed control condition of Dog No. 2 at $\mathrm{Pl} \mathrm{O}$ and $30 \mathrm{cmH}_{2} \mathrm{O}$, respectively. Fig. 2(c) and (d) showed the same lobe in which peribronchial parenchyma was dissected at $\mathrm{Pl} 0$ and $30 \mathrm{cmH}_{2} \mathrm{O}$, respectively. The dissection was limited only into the connective tissues along the bronchi. It took about 30 min to dissect the parenchyma. The leak was checked with soap bubbles by positive bronchial pressure of $30 \mathrm{cmH}_{2} \mathrm{O}$. If there was a leak due to dissection, the lobe was abandoned.

III) The dissected parenchyma was glued to the lobar bronchi in its all surface with aronalpha (Sankyo Co. Japan) at $\mathrm{Pl}$ of $30 \mathrm{cmH}_{2} \mathrm{O}$. This procedure made rigid the glued bronchi not to extend or collapse. All preparations were made at room temperature, $20^{\circ} \mathrm{C}$.

\section{Results}

Pressure-volume $(\mathrm{Pl}-\mathrm{V} l)$ curves of three lobes were shown in Fig. 3. Conditions I, II and III were represented by open circles with continuous line, solid circles with dotted line and triangular marks with interrupted line, respectively. Curves in all conditions were almost the same as those in cases of repeated inflation except volumes in condition III in which $V l$ were slightly decreased due to increased residual volume. The residual volumes of dogs Nos. 1-3 were 65, 50 and $45 \mathrm{ml}$, respectively. Fig. 4 showed $\mathrm{Rl}$ to $\mathrm{Pl}$ relationships of three lobes. Marks of three conditions were the same marks in Fig. 3. Rl's of three conditions were almost the same. $\mathrm{Rl}$ of $\operatorname{dog}$ No. 2 was $1-2 \mathrm{cmH}_{2} \mathrm{O} / \mathrm{liter} / \mathrm{sec}$, while $\mathrm{R} l$ 's of the other dogs were more than $5 \mathrm{cmH}_{2} \mathrm{O} /$ liter/sec. $\mathrm{R} l$ was lowest at $\mathrm{Pl} 5 \mathrm{cmH}_{2} \mathrm{O}$.

Fig. 5 (a) showed MEFVCs of three dogs. In the control curves (left row), 


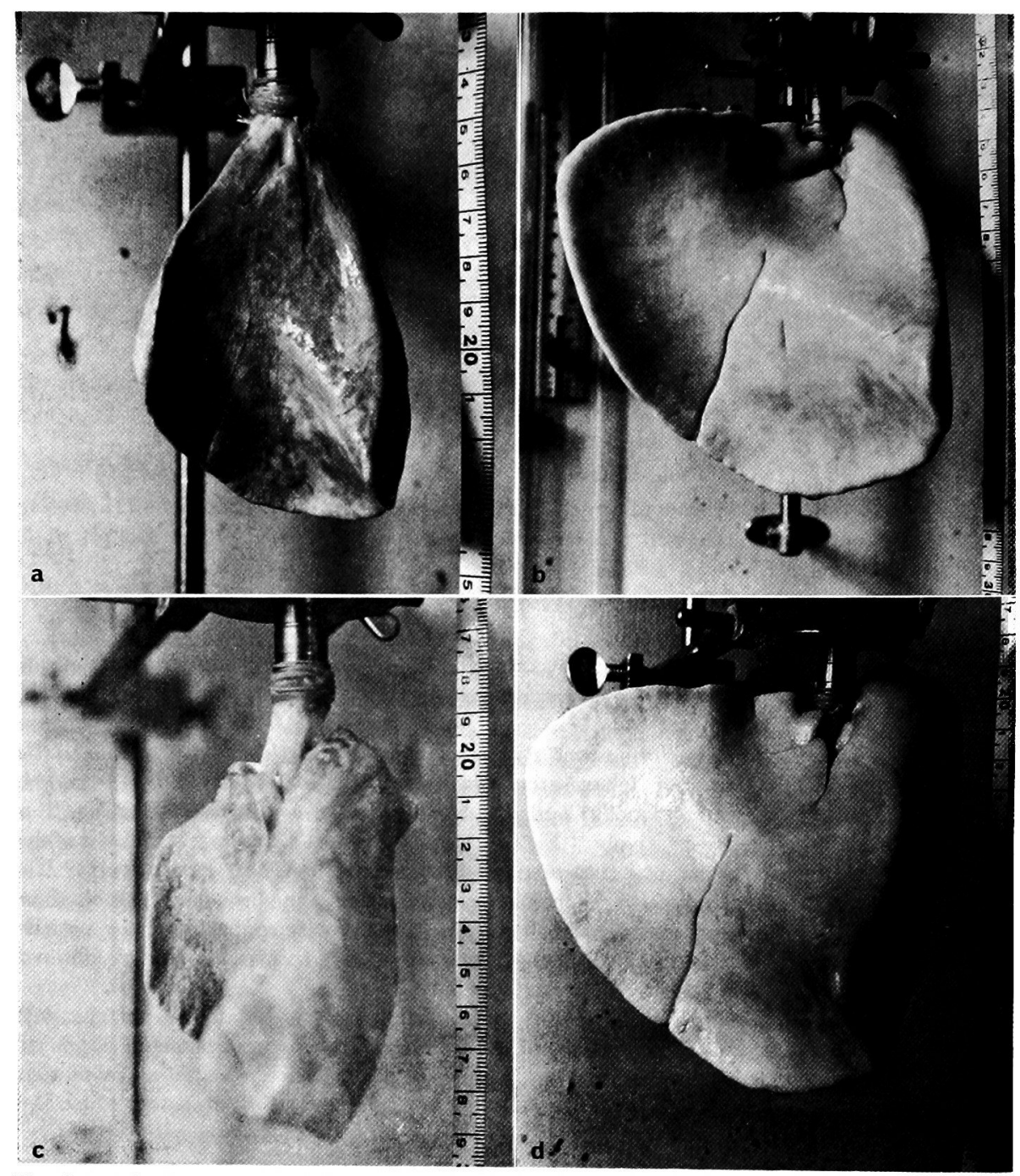

Fig. 2. a, b: condition $\mathrm{I}$ at $\mathrm{Pl} 0 \mathrm{cmH}_{2} \mathrm{O}$ and $\mathrm{Pl} 30 \mathrm{cmH}_{2} \mathrm{O}$, respectively. Extra pulmonary bronchi were partly remained, but very small.

c, d: condition II at $\mathrm{PlO} \mathrm{cmH}_{2} \mathrm{O}$ and $30 \mathrm{cmH}_{2} \mathrm{O}$, respectively.

peak flow was approximately 2.1 liters/sec in dog No. 1, 3.1 liters/sec in dog No. 2 and 1.7 liters/sec in dog No. 3 following almost straight slopes to the end of MEFVC. In condition II (middle row), peak flows were the same or slightly decreased following considerably decreased flows at lower lung volumes, resulting in curve-linear shape toward the volume axis in all dogs. In condition III (right row), every flow became significantly increased except near zero VC. VCs obtained by MEFVCs were almost the same as that obtained from $\mathrm{Pl}$-Vl curves. Comparisons of curves of each dog were also traced on the right row. 


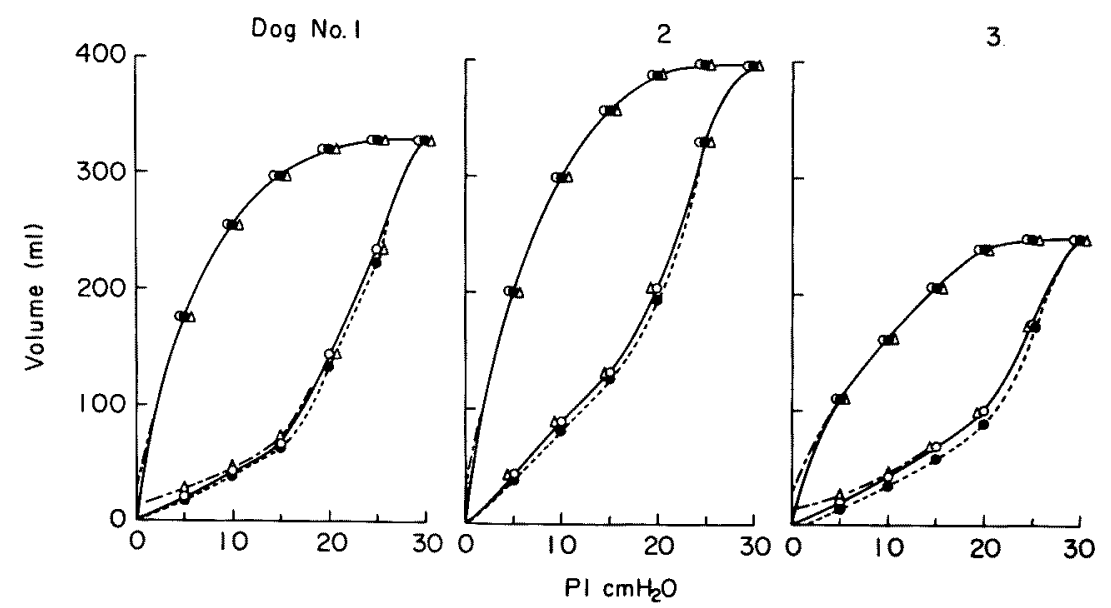

Fig. 3. Lung pressure $(\mathbf{P} l)$-volume $(\mathrm{V} l)$ curves of three conditions. Obtained from three different dogs (Nos. 1, 2, 3). All were almost the same except that $P l$ of condition III was slightly decreased at low lung volumes. O, I; •, II; $\Delta$, III.

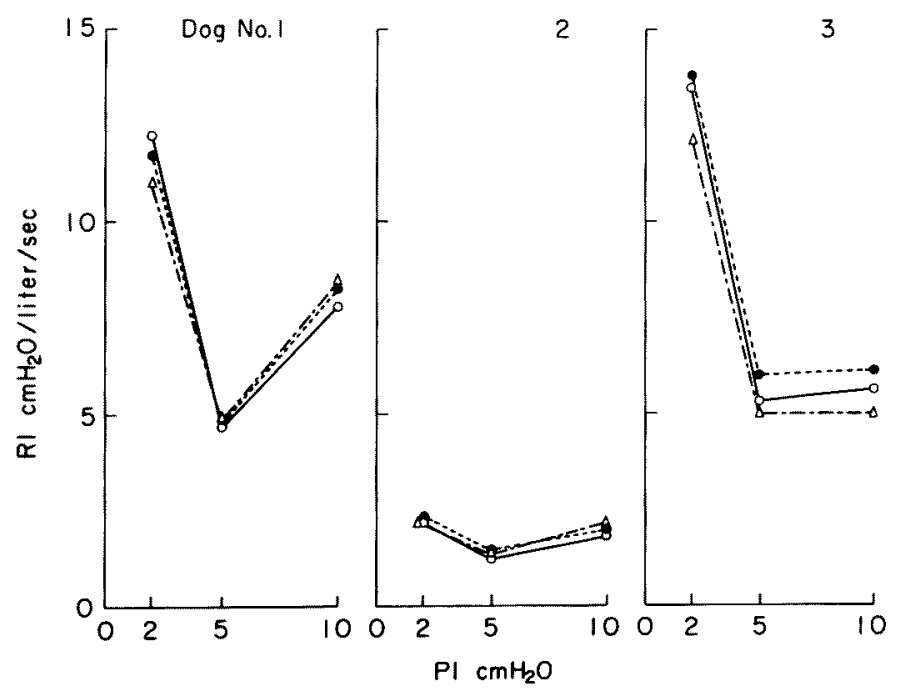

Fig. 4. Airway resistance $(\mathrm{R} l)$ to $\mathrm{P} l$ relationships of three conditions. $\mathrm{R} l$ 's at any $\mathrm{P} l$ of three dogs were almost the same. $\odot, I ; \bullet I I ; \Delta$, III.

Fig. 5(b) showed mean flows of three dogs at 75, 70, 50 and $25 \% \mathrm{VC}$. The peak flow in condition I was standardized as $100 \%$ in each dog. From the mean values flows in three conditions had the same characteristics as mentioned above. The average ratios of $\dot{V}_{\max }$ in condition II to that in condition I were 91, 70 and $40 \%$ at 70,50 and $25 \% \mathrm{VC}$, respectively, and those in condition III to that in condition I were 117,131 and $87 \%$ at the same range of $\mathrm{VC}$, respectively. 


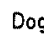

No.
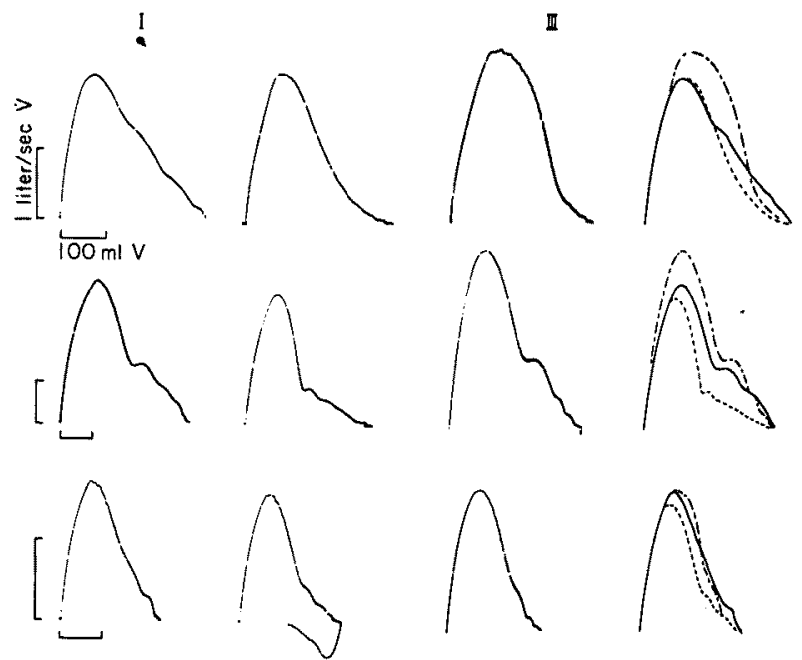

(a)

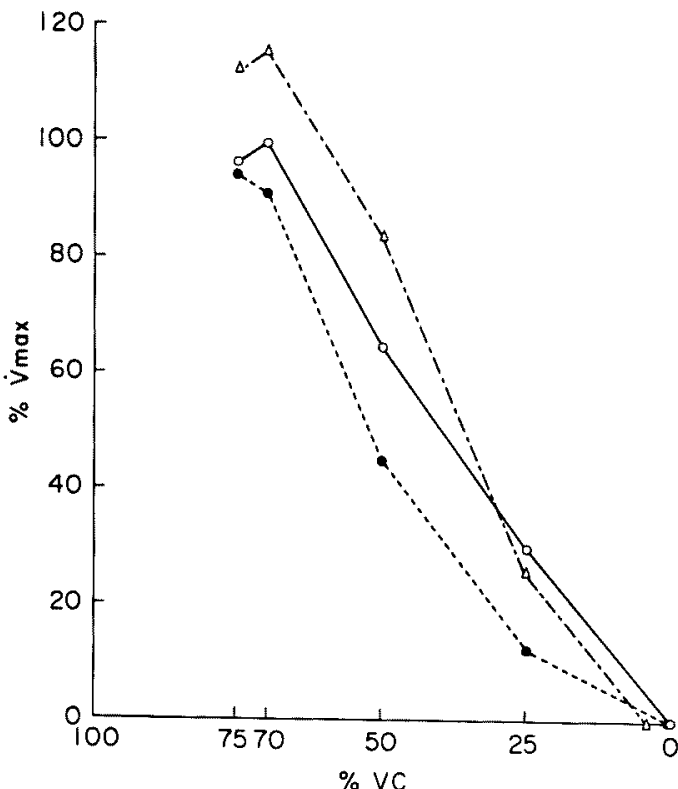

(b)

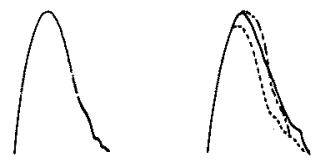

(a)

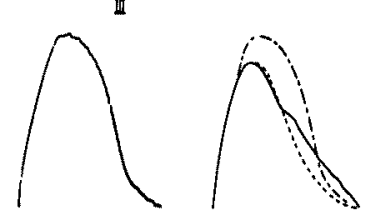


That is, the lung parenchyma attached to the bronchi stretched the bronchial wall radially, making the bronchus hardly collapse. On the contrary, when the bronchi were stored in the parenchyma and became rigid with the glue substance, more flow was obtained. The present study confirmed the static parenchymal effect reported by Takishima et al. (1975) in a dynamic condition.

It was discussed by many investigators that one of the most important factors to limit $\dot{V}_{\text {max }}$ was airway collapse (check valve phenomenon) during maximum expiration (Fry 1958; Pride et al. 1967; Takishima and Sasaki 1972). In intact human lungs Macklem and Wilson (1965) estimated that expiratory airway compression occurred between the segmental bronchi and the glottis at volumes between 75 and $25 \%$ vital capacity and expiratory flow was limited by compression of these airway.

Using a two-dimensional flow model, Takishima and Sasaki (1972) demonstrated that airway collapse occurred in a very limited segment near the outlet of homogeneously collapsible airway indicating stress on the airway to be focussed to a narrow segment, and this might imply that the expiratory check valve occurred over a very limited segment of the bronchi. They also pointed out that $\dot{V}_{\text {max }}$ might be determined by an inverse relationship both of the compliance and of the resistance of the airway from the check valve point to the upstream airway. According to their findings, the site of airway collapse should be determined by the distribution of stiffness of bronchial wall itself times airway resistance from the alveoli to the end of the trachea. And the airway collapse might occur at the site where those products became largest along the airway at maximum expiration.

Macklem and Mead (1968) studied the site of equal pressure point (EPP) along the dog bronchi and found that at high lung volumes EPP was in large extrapulmonary airway and at low lung volumes EPP moved into the intrapulmonary airways. Accordingly, the site of airway collapse in the intact bronchi might start from the proximal of lobar stem-bronchi and move into the more peripheral airway with decreased lung volume. However, in the dissected condition of the present study the dissected segment might contribute to reduce $\dot{V}_{\max }$ all through the $\mathrm{VC}$, and if not, both flows should be the same even in low lung volume. In other words, check valve phenomenon might occur at the dissected segment even at the lower lung volumes due to increased bronchial collapsibility.

Takishima et al. (1975) demonstrated that the parenchymal radial traction on bronchial collapsibility tended to be much intense when the lung tension was increased and the average compliance expressed as collapsing of the airway related to bronchial transmural pressure in an exponential function appeared to be about 75 percent $(55-95 \%)$ of that of the dissected one. Hughes et al. (1974) also reported that interdependence of airway and airspace expansion was less at low lung recoil pressures. In the present study we obtained the opposite results that the effect of parenchymal radial traction was small at high lung volumes; namely, $\dot{V}_{\text {max }}$ of dissected bronchi decreased to $91 \%$ at $75 \% \mathrm{VC}$, while decrease of $\dot{V}_{\max }$ became larger with decreasing lung volumes, and $\dot{V}_{\max }$ decreased to $40 \%$ 
at $25 \% \mathrm{VC}$.

One reason for the opposite effect to $\mathrm{VC}$ might be the difference between dynamic collapse in the present study and static airway collapse examined at constant lung volumes by Takishima et al. (1975). $\dot{V}_{\max }$ at a high lung volume was reported to be effort-dependent (Hyatt et al. 1958), while the effect of bronchial collapse on $\dot{V}_{\max }$ at the effort-dependent part should be limited by the applied driving pressure. In our experiment, larger driving pressure might have made larger difference of $\dot{V}_{\max }$ between conditions I and II. Recently, we observed the effect of lung parenchyma on the dynamic airway collapse and it was demonstrated that the dynamic airway collapse with lung parenchyma was considerably reduced as conferred with that expected from the result statically obtained by Takishima et al. (1975), while there was little dynamic effect on the dissected bronchi (unpublished observation). Accordingly, it is supposed that in the effort-dependent part larger difference between conditions I and II was obtained than that expected from static measurement of airway collapsibility.

In condition III, it was an unexpected result that $\dot{V}_{\max }$ in a low lung volume was slightly lower than the control value. The reason for the inverse effect was not clear but it was supposed that in low lung volume the check valve point might move into upstream, so the rigid bronchial segment would not increase $\dot{V}_{\max }$ any more. On the other hand, residual volumes in condition III were slightly increased; accordingly, it was supposed that at a low lung volume the rigid bronchi with rigidly glued parenchyma might limit the neighboring lung parenchyma to deflate their volume freely, resulting in increased residual volume and limited $\dot{V}_{\max }$. Several models have been reported which analyzed the effect of airway collapsibility on the MEFVC. Blank et al. (1969) reported that $\dot{V}_{\max }$ was increased by stinting the trachea in the dog lung and suggested that effect of tracheal collapsibility on $\dot{V}_{\max }$ was also intense owing to fixation of check valve point to the more collapsible trachea. Pardaens et al. (1972) made a computer analysis on a model simulating the pressure-flow-volume relationship during expiration, and showed that elastic properties of the airways primarily determined $\dot{V}_{\mathrm{max}}$. However, their analysis on the MEFVC was based on the pressure-radius relationships of the dissected bronchi or extrapulmonary airways, and they payed no attention to the effect of lung parenchyma.

Then a question to arise is how the lung parenchyma influences on MEFVC; in other words, what kind of model is more suitable for analysis. Lambert and Willson (1973) made a mathematical analysis of parenchymal effect on airway deformation and presumed that the presence of the parenchyma reduced the change in airway radius with pressure by as much as $50 \%$ at a high lung volume. Caldwell (1971) examined the experimentally induced emphysema in the rabbit lung, and reported that alveolar attachment of non-cartilaginous airways was significantly decreased in papain treated lungs and the maximal expiratory flow was lower. This indicated that in spite of no significant differences of elastic recoil between the papain and the control lungs the dynamic performance of the 
papain lung was decreased owing to loss of the radial stresses of alveolar walls to airways, rendering them unstable at normal expiratory flow level rather than a change in the physical properties of the airway wall. Caldwell and Fiy (1969) also suggested that the upper portion of the MEFVC was reduced owing to cartilaginous airways and the lower portion to non-cartilaginous airways. We dissected very limited stem of lobar bronchi without any deformation of the peripheral bronchi and it was limited to the parenchymal destruction without either $\mathrm{P} l$ change or any airway resistance change. Using EPP theory, Mead et al. (1967) demonstrated that $\dot{V}_{\max }$ was determined by $\mathrm{Pl}$ devided by upstream resistance. However, in the present experimental conditions the airway collapsibility at check valve point would be only the limiting factor of $\dot{V}_{\max }$ because any changes of $\mathrm{Pl}$ and peripheral airway resistance were not introduced.

Maisel et al. (1968) studied airway dynamics of 38 excised postmortem human lungs and revealed in 26 cases that expiratory dynamic collapse of medium bronchi was associated with distally diffuse or zonally localized atrophy of the bronchial wall. It tended to coexist with emphysema, a loss of radial support from the peribronchial tissue, and its contribution to airway obstruction was greatly increased when emphysema was present. Accordingly, although the present model could not directly be applied to the real obstructive lung disease, it was one of the very simple and useful models for the study of the effect of parenchymal radial stress on the bronchi.

\section{References}

1) Blank, N., Dilley, R. \& Nadel, J.A. (1969) Increase in expiratory airflow by stinting the trachea. Physiologist, 12, 177.

2) Caldwell, E.J. (1971) Physiologic and anatomic effects of papain on the rabbit lung. J. appl. Physiol., 31, 458-465.

3) Caldwell, E.J. \& Fry, D.L. (1969) Pulmonary mechanics in the rabbit. J. appl. Physiol., 27, 280-285.

4) Fry, D.L. (1958) Theoretical considerations of the bronchial pressure-flow-volume relationships with particular reference to the maximum expiratory flow volume curve. Phys. in Med. Biol., 3, 174-194.

5) Goldman, M., Knudson, R.J., Mead, J., Peterson, N., Schwaber, J.R. \& Whol, M.E. (1970) A simplified measurement of respiratory resistance by forced oscillation. J. appl. Physiol., 28, 113-116.

6) Hughes, J.M.B., Jones, H.A., Wllson, A.G., Grant, B.J.B. \& Pride, N.B. (1974) Stability of intrapulmonary bronchial dimensions during expiratory flow in excised lungs. J. appl. Physiol., 37, 684-694.

7) Hyatt, R.E., Schilder, D.P. \& Fry, D.L. (1958) Relationship between maximum expiratory flow and degree of lung inflation. J. appl. Physiol., 13, 331-336.

8) Lambert, R.K. \& Willson, T.A. (1973) A model for the elastic properties of the lung and their effect on expiratory flow. J. appl. Physiol., 34, 34-48.

9) Macklem, P.T. \& Mead, J. (1968) Factors determining maximum expiratory flow in dogs. J. appl. Physiol., 25, 159-169.

10) Macklem, P.T. \& Wilson, N.J. (1965) Measurement of intrabronchial pressure in man. J. appl. Physiol., 20, 653-663.

11) Maisel, J.C., Silvers, G.W. \& Mitchell, R.S. (1968) Bronchial atrophy and dynamic expiratory collapse. Amer. Rev. resp. Dis., 98, 988-997. 
12) Mead, J., Turner, J.M., Macklem, P.T. \& Little, J.B. (1967) Significance of the relationship between lung recoil and maximum expiratory flow. J.appl. Physiol., 22, 95-108.

13) Mead, J., Takishima, T. \& Leith, D. (1970) Stress distribution in lungs, a model of pulmonary elasticity. $J$. appl. Physiol., 28, 596-608.

14) Pardaens, J., Van De Woestijne, K.P. \& Clement, J. (1972) A physical model of expiration. J. appl. Physiol., 33, 479-490.

15) Pride, N.B., Permitt, S., Riley, R.L. \& Bromberger-Barnea, B. (1967) Determinants of maximum expiratory flow from the lungs, J. appl. Physiol., 23, 646-662.

16) Takishima, T. \& Sasaki, H. (1972) Two-dimensional flow-model for analysis of expiratory check valve. Bull. Physio-path. resp., 8, 361-374.

17) Takishima, T., Sasaki, H. \& Sasaki, T. (1975) Influence of lung parenchyma on collapsibility of dog bronchi. J. appl. Physiol., 38, 875-881. 